\title{
Chronic histological outcomes of indirect traumatic optic neu- ropathy in adolescent mice: Persistent degeneration and tempo- rally regulated glial responses.
}

\author{
Shelby M. Hetzer ${ }^{1}$, Emily M. Shalosky ${ }^{2}$, Jordyn N. Torrens ${ }^{3}$, and Nathan K. Evanson 1,3,4,* \\ 1 University of Cincinnati College of Medicine, Neuroscience Graduate Program \\ 2 University of Cincinnati, Department of Biological Sciences \\ 3 Cincinnati Children's Hospital Medical Center, Division of Pediatric Rehabilitation Medicine \\ 4 University of Cincinnati, Department of Pediatrics \\ * Correspondence: Nathan.evanson@cchmc.org;
}

\begin{abstract}
Injury to the optic nerve, termed, traumatic optic neuropathy (TON) is a known comorbidity of traumatic brain injury (TBI) and is now known to cause chronic and progressive retinal thinning up to 35 years after injury. Although animal models of TBI have described the presence of optic nerve degeneration and research exploring acute mechanisms is underway, few studies in humans or animals have examined chronic TON pathophysiology outside the retina. We used a closed-head weight-drop model of TBI/TON in 6-week-old male C57BL/6 mice. Mice were euthanized 7-, 14-, 30-, 90-, and 150-days post injury (DPI) to assess histological changes in the visual system of the brain spanning a total of 12 regions. We show chronic elevation of FluoroJade-C, indicative of neurodegeneration, throughout the time course. Intriguingly, FJ-C staining revealed a bimodal distribution of mice indicating the possibility of subpopulations that may be more or less susceptible to injury outcomes. Additionally, we show that microglia and astrocytes react to optic nerve damage in both temporally and regionally different ways. Despite these differences, astrogliosis and microglial changes were alleviated between 14-30 DPI in all regions examined, perhaps indicating a potential critical period for intervention/recovery that may determine chronic outcomes.
\end{abstract}

Keywords: traumatic optic neuropathy; head trauma; adolescent head trauma; mice; chronic; gliosis; neurodegeneration

\section{Introduction}

An estimated $43 \%$ of traumatic brain injury (TBI) survivors will develop chronic impairment (e.g., migraine, motor impairment, memory decline, visual deficits, etc.), amounting to roughly three million long-term disability cases in the US [1-3]. Roughly 812,000 of these cases occur in children and adolescents under 17 each year (CDC), making TBI the leading cause of death and disability among this age group [4]. Thus, it is necessary to understand how chronic symptoms develop, how long they take to develop, and how long they may persist, to inform appropriate treatment of traumatic brain injury in adolescents. A major injury mechanism in TBI is traumatic axonal injury (TAI), which results from shearing acceleration/deceleration forces of impact or compaction of white matter tracts. Chronic white matter damage and gliosis are also major drivers of disability after TBI [5], and TAI is associated with worse psychological and behavioral outcomes in adolescents [6].

Among animal models for TAI, injury to the optic nerve/tract has been reported (e.g., [7-10]), making it an attractive approach for the study of optic nerve injury due to its wellcharacterized anatomy. Examination of optic nerve injury through a TAI model is also important because visual impairment occurs in roughly $20 \%$ of children with TBI [11]. We previously described an adolescent murine closed-head trauma model characterized by 
axonal degeneration and gliosis in the optic nerve and its projection targets, retinal ganglion cell death, and behavioral visual impairment [12-14]. This injury model appears to produce damage to the optic nerve via force transmission through the optic canal and reproduces key features of indirect traumatic optic neuropathy (iTON). iTON is a chronic TBI-associated problem, and there is evidence of increased retinal deterioration and worsening of visual impairments for at least 35 years after TBI [15]. These findings in optic nerve injury patients parallel rat experimental TBI, in which there is persistent white matter thinning at least six months after injury. Diffusion weighted/tensor imaging of TAI patients also reveals long-term decline in white matter pathology at least 8 months after injury [16].

This chronic worsening of axonal pathology is likely due to Wallerian Degeneration (WD), an active degenerative process which is characteristically slower in the central nervous system than the peripheral nervous system(i.e., days vs. months to years) likely due to glial cell activity [17]. WD mechanisms have been well described and reviewed with the general pattern of degeneration proceeding as follows: (1) axon-specific injury, (2) distal axon degeneration, mitochondrial dysfunction, and calcium release, and (3) microglial/oligodendrocyte response (e.g.[18]; reviewed in $[17,19,20])$. Though WD in the CNS can occur for days to years following axonal injury, follow-up in many studies ends well before potential onset of chronic pathology.

Interestingly, diffusion tensor imaging studies show the potential for recovery of white matter tracts 30 days after TBI [21]. This potential for recovery is supported through axonal reorganization/recovery in the optic system in rodent models of TAI, although it arises a bit earlier (2-14 days after optic nerve injury) [22-24]. Differences in the time course of TAI/WD progression likely vary depending on injury methodologies, the lengths of axons injured, and the presence of cell death. For example, TAI could result in initial cell death and axonal dieback followed by (a) brief recovery of axonal integrity and subsequent decline [25] or (b) continued improvement [18]. Alternatively, TAI (including cases of iTON) is associated with delayed and progressive worsening of axonal injury due to processes of secondary WD via recruitment of glial cells and activation of immune responses in the presence of axonal debris [26]. In support of this, iTON patients showed delayed presentation of symptoms up to 7-9 years after injury [27], which could indicate unknown early protective or reparative mechanisms of the optic nerve that are not sustainable after TAI particularly in cases where cell death occurs (i.e., retinal cell death).

Thus, examining the long-term histological effects and cellular responses of degeneration following optic nerve injury could improve understanding of diagnosis and treatment of iTON and/or TAI following brain injury. Accordingly, we undertook a long-term follow up study of the brain histologic response to optic nerve injury after blunt head trauma, in primary and secondary targets of optic nerve projections in adolescent mice. We hypothesized that there would be chronic degeneration of the visual system following head trauma that would follow a pattern of axonal Wallerian degeneration and persist long after initial insult.

\section{Materials and Methods}

\subsection{Animals}

Experiments were performed in 6-week old adolescent male C57BL/6J mice (Jackson Laboratories, Bar Harbor, $\mathrm{ME}$ ). Mice were housed under a 14h:10h light:dark schedule in pressurized individually ventilated cage racks, with 4 mice per cage, and were given ad libitum access to water and standard rodent chow. Animals habituated to the vivarium for one week prior to undergoing traumatic brain injury and subsequent procedures. The University of Cincinnati Institutional Animal Care and Use Committee approved all experimental procedures (protocol \# 20-02-26-02, approved 4/16/2020).

\subsection{Traumatic Brain Injury}

Closed-head injury was performed by weight drop, as previously described [12,28]. Briefly, mice were anesthetized using isoflurane (2-3\%) until no toe-pinch reflex was present and placed in prone position under a metal rod (1.2 $\mathrm{cm}$ diameter; $400 \mathrm{~g}$ ) that was raised to $1.5 \mathrm{~cm}$ above the intact, unshaven scalp. The head was rested on a $0.5 \mathrm{~cm}$ thick piece of corkboard to prevent head displacement and reduce acceleration/deceleration of the weight force. Injury was produced by dropping the rod over the approximate location of bregma. After head trauma, mice were placed under an oxygen hood made from a pipet box connected to an oxygen tank and were given $100 \%$ oxygen $\left(\mathrm{O}_{2}\right)$ until normal breathing returned - approximately 1-5 minutes. Mice were then removed from the chamber and, upon regaining righting reflex, were returned to their home cages. Injured mice will hereon be referred to as TBI mice. 
Sham animals were anesthetized, weighed, and allowed to recover. Initially, 84 mice underwent the aforementioned procedure and were divided in cohorts of sham and TBI for five chronic time points -7 (sham $\mathrm{n}=8$, TBI $\mathrm{n}=8), 14$ (sham $n=8, T B I n=8), 30$ (sham $n=8$, TBI $n=8), 90$ (sham $n=8$, TBI $n=8)$, and 150 (sham $n=10, T B I n=10)$ days post injury (DPI). Unfortunately, after initial procedures, four of the eight TBI mice for the 30 DPI time-point were lost, so a new set of eight sham and eight TBI mice were run separately following identical procedures and added to the remaining cohort. See Figure 1 for a timeline for each cohort.

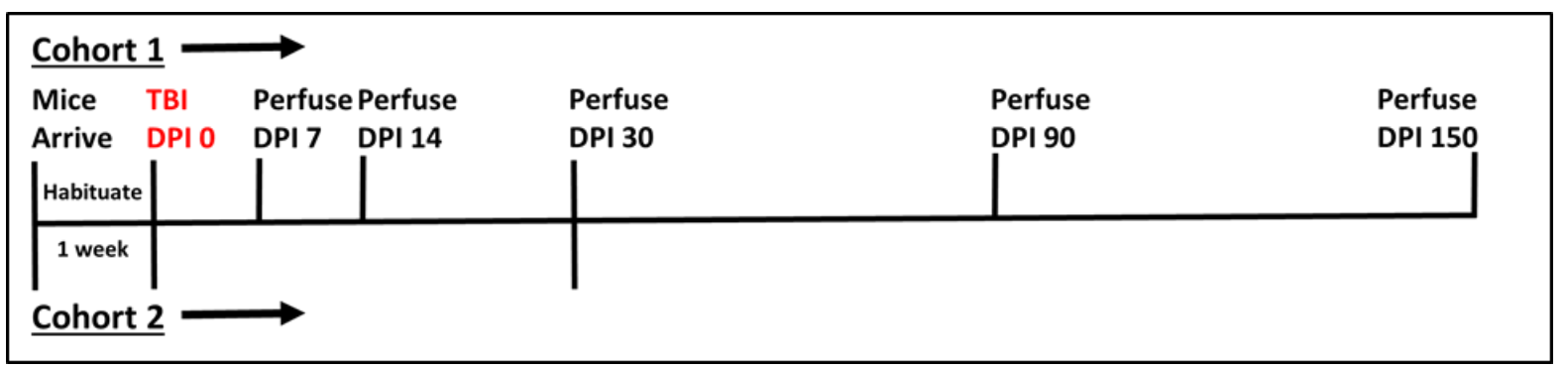

Figure 1. Timeline of experimental procedures. Cohort 1 was our initial experimental cohort with all mice experiencing TBI on the same Day (DPI 0), and subgroups perfused at each timepoint noted. Due to loss of some tissue samples from cohort 1, however, a second cohort (Cohort 2) was added to the 30 DPI time point. Vertical Lines above the horizontal indicate Cohort 1 while those below the horizontal line are associated with Cohort 2.

\subsection{Histology}

\subsubsection{Tissue Collection}

For immunohistochemical (IHC) analysis, mice were euthanized using Fatal Plus ${ }^{\circledR} 7,14,30,90$, and 150 DPI. Mice were perfused transcardially with $4 \%$ paraformaldehyde in $0.02 \mathrm{M}$ phosphate-buffered saline (PBS) solution ( $\mathrm{pH}$ 7.4). Brains were removed and post-fixed in $4 \%$ paraformaldehyde in $0.01 \mathrm{M}$ PBS, rinsed in $0.01 \mathrm{M}$ PBS, and immersed in 30\% sucrose solution at $4^{\circ} \mathrm{C}$ until sectioning. Sucrose-saturated brains were frozen on dry ice and sectioned at $35 \mu \mathrm{m}$ using a sliding microtome (Leica, Bannockburn, IL). Sections were stored in cryoprotectant solution (0.01M PBS, polyvinylpyrrolidone (Sigma -Cat\# PVP-40), ethylene glycol (Fisher Cat \# E178-4), and sucrose (Fisher Cat \# S5-3)) at $-20^{\circ} \mathrm{C}$ until staining or IHC was performed.

Fluoro-Jade C (FJ-C; Histo-Chem, Jackson, AR; cat\# 1FJC), a marker for degenerating neurons and axons [29], was used to stain tissue sections according to the manufacturer's directions. Briefly, sections were flat-mounted on positively charged slides, washed, incubated in $0.06 \%$ potassium permanganate for 5 minutes, washed, incubated in $0.0001 \% \mathrm{FJ}-$ $\mathrm{C}$ solution (diluted in $1 \%$ acetic acid) for 5 minutes. After staining, slides air dried in the dark. Slides were stored without coverslips in a slide box and imaged immediately to reduce background severity.

\subsection{Immunofluorescence}

Primary antibodies used for immunofluorescence were polyclonal rabbit anti-glial fibrillary acidic protein antibody (GFAP 1:2000; DAKO, Santa Clara, CA; cat \# Z0334; RRID AB_10013382) and ionized calcium-binding adaptor molecule 1 (Iba-1 1:2000; Synaptic systems, Goettingen, Germany; cat\# 234003, RRID AB_10641962), both at 1:2000 dilution (Table 1). As discussed previously, increased GFAP immunoreactivity was measured as an indicator of astrogliosis; IBA-1 labels microglia; soma area and perimeter were used as measures of changing morphology associated with microglial activation [13]. For detailed procedures, see Hetzer, et al., 2021. Table 1 shows more detailed antibody information.

\subsection{Image Analysis}

GFAP and IBA-1 slides were photographed using an Axio lmager Z1 microscope with an Apotome (Leica Microsystems, Buffalo Grove, IL). All slides were photographed using the same exposure and magnification between treatment conditions within each time point. FJ-C slides were imaged on a Nikon C2 Plus Confocal Microscope (Nikon Corporation, Melville, New York) at identical exposure and fluorescence intensity/color settings so that accurate mean fluorescence could be compared between groups. A blinded observer took all pictures. Image analysis and quantification of mean fluorescence intensity was also performed by a blinded investigator using ImageJ software [30] for GFAP and FJ-C. IBA-1 cell soma area and perimeter were measured using Nikon Elements Analysis software (Nikon, Melville, NY). 


\begin{tabular}{|c|c|c|c|c|c|}
\hline Antibody & RRID & $\begin{array}{c}\text { Host } \\
\text { Species }\end{array}$ & Immunogen & Manufacturer & Concentration \\
\hline GFAP & AB_10013382 & Rabbit & $\begin{array}{l}\text { Whole bovine } \\
\text { GFAP, isolated } \\
\text { from spinal cord. }\end{array}$ & $\begin{array}{c}\text { DAKO (Agilent) } \\
\text { CAT: Z033401-2 } \\
\text { Lot: } 00059585\end{array}$ & $1: 2000$ \\
\hline Iba-1 & AB_10641962 & Rabbit & $\begin{array}{l}\text { Peptide from c- } \\
\text { terminal sequence } \\
\text { from rat Iba-1: } \\
\text { PTGPPAK } \\
\text { KAISELP }\end{array}$ & $\begin{array}{c}\text { Synaptic } \\
\text { Systems } \\
\text { CAT: } 234003 \\
\text { Lot: } 234003 / 9\end{array}$ & $1: 2000$ \\
\hline $\begin{array}{c}\text { Cy3 } \\
\text { AffiniPure } \\
\text { Donkey anti } \\
\text { Rabbit IgG } \\
\text { (H+L) } \\
\text { conjugated } \\
\text { secondary }\end{array}$ & AB_2307443 & Donkey & $\begin{array}{c}\text { Gamma } \\
\text { immunoglobulins, } \\
\text { heavy and light } \\
\text { chains }\end{array}$ & $\begin{array}{c}\text { Jackson } \\
\text { Immuno } \\
\text { Research } \\
\text { CAT: } 711-165- \\
152 \\
\text { Lot: } 79424\end{array}$ & $1: 500$ \\
\hline
\end{tabular}

\subsection{Statistical analysis}

We performed statistical analysis using the SigmaPlot 14 software package (Systat, San Jose, CA). Alpha was set at 0.05. Within individual analyses, where $\mathrm{n}$ did not equal that reported in the cohort numbers above, this was due to loss of tissue, inability to measure fluorescence due to background, removal of a significant outlier (Grubbs test for outliers p <0.05; https:/www.graphpad.com/quickcalcs/Grubbs1.cfm), or the addition of the second 30 DPI cohort. Occasionally, if a significant outlier was found, and its removal from the analysis resulted in no change in statistical outcome, it was retained on the graph for the sake of transparency (e.g. Fig. 5a 14 DPI sham). Weight data were first normalized as percent of pre-injury weight, then analyzed using 2-way repeated measures ANOVA (with injury and time after injury as independent variables). Data are reported as mean and SEM, except where specified otherwise. Post-hoc testing was performed using the Holm-Sidak post-hoc method. Because we did not plan to compare time points and because histologic staining was performed for each time point separately, we did not compare directly between time points. We analyzed planned comparisons with student's t tests within each time point and reported as mean and SEM in figures. If normality testing failed, Mann-Whitney Rank Sum Tests were used, and those data are reported as median and interquartile range (see tables 2-4 for test statistics).

After analysis of FJ-C, we noted that FJ staining in TBI groups did not always appear normally distributed for several regions of interest (ROIs) examined. Therefore, we performed post-hoc analyses for normality (i.e., ShapiroWilk normality tests and Kolmogorov-Smirnov tests) and graphed the frequency distributions whenever normality failed. After confirming a bi-modal distribution, we split TBI groups into high-staining and low-staining mice using a median split. We then used student's $t$ tests between these groups and compared low and high to sham. While we also noted distributions in a few ROIs for GFAP and IBA-1 that appeared similar to these bimodal groups by visual inspection, these analyses did not fail tests for normality, and so were not included.

\section{Results}

\subsection{Weight, Righting, and Seizures}

Mortality was low with only 4 out of 88 mice dying after injury (cause of death appeared to be prolonged apnea, as we have previously reported [13]). There were no skull fractures or other overt physical injuries on or inside the skull. Pre-injury, mice weighed between 18.1 and 24.2g (M=20.97, SD=1.31) and weights between groups were not significantly

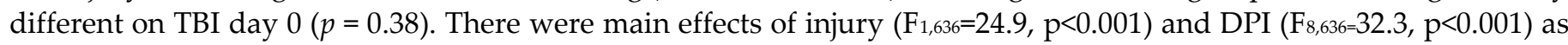
well as an interaction $\left(\mathrm{F}_{8,636}=2.2, \mathrm{p}=0.03\right)$. Post hoc analyses revealed significantly lower weights in the TBI group at all time-points after injury except 11 and 16 DPI (sup. fig. 1a). Righting times for TBI mice ranged from 1.17 to 21.16 minutes $(\mathrm{M}=5.26, \mathrm{SD}=4.02)$. Starting weight did not correlate with righting time after injury $(\mathrm{r}=0.04, p=0.81$; sup. fig. $1 \mathrm{~b})$. Some mice showed overt signs of convulsions/generalized tonic-clonic seizures after injury, but these did not correlate with starting weight $(\mathrm{r}=-0.2, p=0.22$; sup. fig. $1 \mathrm{c})$ or righting time $(\mathrm{r}=0.18, p=0.25$; sup. fig. $1 \mathrm{~d})$.

\subsection{Histology}

We examined several regions of interest throughout the cortex, diencephalon, tectum, and brainstem for signs of degeneration and gliosis utilizing FJ-C (statistics in Table 2), GFAP (statistics in Table 3), and IBA-1 (statistics in Table 
4) staining. For this study, regions of interest were limited to the visual/optic system. Regions included the optic tract (OT) and its direct projections the dorsal lateral geniculate nucleus (dLGN), ventral lateral geniculate nucleus (vLGN), suprachiasmatic nucleus (SCN), superior colliculi (SC), nuclei of the accessory optic tract - the dorsal (DTN) and medial terminal nuclei (MTN) - the accessory optomotor nucleus (AON), and Edinger-Westphal nuclei (EW). We also investigated multi-synaptic targets including the brachium of the superior colliculus ([BoSC] also includes some direct OT axons), nucleus of the optic tract (NOT), visual cortex (VC), and regions that integrate motor coordination of vision the substantia nigra pars reticulata and caudoputamen (see supplementary figures 2 and 3 for anatomy). For more information on the anatomy of these regions and justification of our choices see: Giolli, et al. for the accessory optic system [31]; other regions controlling eye movements [32,33]; comprehensive retinofugal projections [34]; superior colliculi division [35]; visuo-motor circuit [36,37]; and NOT [38]. Outside these regions, there was somatic staining only in the hippocampus, which we have reported previously [13].

\section{Table 2. Statistics for IHC - Fluoro Jade-C}

\begin{tabular}{|c|c|c|c|c|c|c|c|c|}
\hline Time Point & OT & $p$ & dLGN & $p$ & vLGN & $p$ & SC & $p$ \\
\hline 7 DPI & $t(14)=-16.94$ & $\mathrm{p}<0.001$ & $t(14)=-4.04$ & $\mathrm{p}<0.001$ & $t(14)=-5.17$ & $\mathrm{p}<0.001$ & $t(11)=-12.46$ & $\mathrm{p}<0.001$ \\
\hline 14 DPI & $t(13)=-11.08$ & $\mathrm{p}<0.001$ & $\mathrm{U}(13)=0$ & $\mathrm{p}<0.001$ & $\mathrm{U}(13)=0$ & $\mathrm{p}<0.001$ & $\mathrm{U}(13)=0$ & $\mathrm{p}<0.001$ \\
\hline 30 DPI & $\mathrm{U}(26)=18$ & $\mathrm{p}<0.001$ & $t(14)=-4.78$ & $\mathrm{p}<0.001$ & $U(25)=-39.5$ & $\mathrm{p}=0.02$ & $t(26)=-4.12$ & $\mathrm{p}<0.001$ \\
\hline 90 DPI & $t(13)=-16.57$ & $\mathrm{p}<0.001$ & $t(14)=-0.77$ & $\mathrm{p}=0.05$ & $t(13)=-2.47$ & $\mathrm{p}=0.03$ & $t(12)=-6.21$ & $\mathrm{p}<0.001$ \\
\hline 150 DPI & $t(19)=6.74$ & $\mathrm{p}<0.001$ & $U(15)=26$ & $\mathrm{p}<0.001$ & $t(15)=0.79$ & $\mathrm{p}=0.44$ & $t(15)=-5.60$ & $p=0.002$ \\
\hline & DTN & $p$ & MTN & $p$ & BoSC & $p$ & NOT & $p$ \\
\hline 7 DPI & $\mathrm{U}(15)=0.0$ & $\mathrm{p}=0.004$ & $t(14)=-3.44$ & $p=0.004$ & $t(14)=-4.69$ & $\mathrm{p}<0.001$ & $\mathrm{U}(13)=22$ & $\mathrm{p}=0.54$ \\
\hline 14 DPI & $\mathrm{U}(14)=0$ & $\mathrm{p}<0.001$ & $\mathrm{U}(12)=0$ & $\mathrm{p}=0.001$ & $t(13)=-4.00$ & $\mathrm{p}=0.001$ & $t(13)=-3.27$ & $\mathrm{p}=0.006$ \\
\hline 30 DPI & $\mathrm{U}(15)=4$ & $\mathrm{p}=0.002$ & $\mathrm{U}(15)=2$ & $\mathrm{p}<0.001$ & $\mathrm{U}(13)=14$ & $\mathrm{p}=0.12$ & $\mathrm{U}(24)=43$ & $\mathrm{p}=0.04$ \\
\hline 90 DPI & $t(13)=-4.95$ & $\mathrm{p}<0.001$ & $\mathrm{U}(14)=0$ & $\mathrm{p}<0.001$ & $t(12)=-5.09$ & $\mathrm{p}<0.001$ & $t(13)=-3.28$ & $\mathrm{p}=0.006$ \\
\hline 150 DPI & $t(14)=0.02$ & $p=0.02$ & $\mathrm{U}(17)=38$ & $\mathrm{p}=0.89$ & $t(17)=-5.76$ & $\mathrm{p}<0.001$ & $\mathrm{U}(19)=19$ & $p=0.02$ \\
\hline
\end{tabular}

Table 2. Test statistics for histological FJ-B data. $t$ indicates a student's t test; U indicates that data failed normality and a Mann Whitney Rank-Sum analysis was performed; p represents p-values of analyses. Abbreviations: OT: Optic Tract; dLGN: dorsal Lateral Geniculate Nucleus; vLGN: ventral LGN; SC: Superior Colliculi; DTN: Dorsal Terminal Nucleus; MTN: Medial Terminal Nucleus; BoSC: Brachium of the Superior Colliculi; NOT: Nucleus of the Optic Tract.

Optic Tract. There was significantly increased FJ-C staining in injured mice at all five time points (fig. 2a-c; table 2). At 30 DPI we saw high and low-expression of FJ-C in TBI mice. We confirmed a bi-modal distribution within the TBI group at 30 DPI (fig. 3a) and found significant differences between these groups ( $p<0.001$; fig. 3b). The low expressing FJ mice still presented with significantly higher FJ than sham ( $p=0.008$; fig $3 c)$ as well as high expressers versus sham ( $p<0.001$; sup. fig. 3d). Increased GFAP immunofluorescence (i.e., astrogliosis) was present in the optic tract 7, 14, 90, and 150 DPI (fig. 4a-c; table 3; sup. fig. 3b). Microglial IBA-1 staining followed the same pattern as FJ-C with significantly larger somata in the OT at all time-points after injury in TBI mice (fig. 5a-c; table 4; sup. fig. 3c). We also measured soma perimeter, which produced nearly identical results for this and subsequent ROIs (data reported in sup. table 1).

Lateral Geniculate Nucleus. In the dorsal LGN there was increased axonal degeneration 7, 14, 30, and 90 DPI (sup. fig. 4a, d; table 2). There was no longer a significant difference between sham and TBI mice 150 DPI; however, there is 
a significant difference between high and low expression in injured mice ( $\mathrm{p}=0.005$; sup. fig. 5i, 1 ) and low FJ-expressing mice were no longer different than sham ( $p=0.2$; sup. fig. 5k). Despite overall increases in FJ in the TBI group 30 and 90 DPI, the TBI group significantly split 30 DPI (p<0.001; sup. fig. 5a,b) and 90 DPI (p<0.001; sup. fig 5e,f), but low FJexpressing mice were only not different from sham $90 \mathrm{DPI}(\mathrm{p}=0.8$; sup. fig. $5 \mathrm{~g}$ ). In the ventral LGN, there was significant degeneration in injured mice 7, 14, 30, and 90. DPI 150 was not significantly different between groups (sup. fig. 6a, d), and, again high and low expressers were significantly different from each other $(p=0.006$; sup. fig. 7i, j), low was similar to sham ( $p=0.1$; sup. fig. $7 \mathrm{k})$, and high was significantly higher than sham ( $\mathrm{p}=0.01$; sup. fig. 7l). This split also occurred 30 DPI ( $p=0.004$; low vs sham $p=0.8$; high vs sham $p<0.001$; sup. fig. 7a-d) and 90 DPI ( $<<0.001$; low vs sham $p=0.56$; high vs sham $\mathrm{p}=0.001$ ); sup. fig. 7e-h). Additionally, degeneration is not different between the dLGN and vLGN at any time point (sup. table 2).

\section{Table 3. Statistics for IHC - GFAP}

\begin{tabular}{|c|c|c|c|c|c|c|c|c|}
\hline Time Point & OT & $p$ & dLGN & $p$ & vLGN & $p$ & SCN & $p$ \\
\hline 7 DPI & $t(14)=5.27$ & $\mathrm{p}<0.001$ & $t(14)=-1.56$ & $\mathrm{p}=0.14$ & $t(14)=0.12$ & $\mathrm{p}=0.90$ & $t(13)=1.02$ & $\mathrm{p}=0.33$ \\
\hline 14 DPI & $t(13)=-2.65$ & $\mathrm{p}=0.02$ & $t(13)=1.13$ & $\mathrm{p}=0.28$ & $t(13)=1.25$ & $\mathrm{p}=0.23$ & $t(13)=1.11$ & $p=0.29$ \\
\hline 30 DPI & $t(14)=2.07$ & $\mathrm{p}=0.07$ & $t(14)=-4.00$ & $\mathrm{p}=0.001$ & $t(14)=-3.64$ & $\mathrm{p}=0.003$ & $t(13)=-2.28$ & $\mathrm{p}=0.25$ \\
\hline 90 DPI & $t(14)=-3.91$ & $\mathrm{p}=0.002$ & $t(14)=-1.76$ & $\mathrm{p}=0.10$ & $t(14)=-1.09$ & $\mathrm{p}=0.43$ & $t(14)=1.78$ & $\mathrm{p}=0.10$ \\
\hline \multirow[t]{2}{*}{150 DPI } & $t(17)=-4.49$ & $\mathrm{p}<0.001$ & $t(18)=2.51$ & $\mathrm{p}=0.02$ & $t(18)=3.71$ & $\mathrm{p}=0.002$ & $t(18)=2.51$ & $\mathrm{p}=0.02$ \\
\hline & SC & $p$ & DTN & $p$ & MTN & $p$ & BoSC & $p$ \\
\hline 7 DPI & $t(13)=-4.12$ & $\mathrm{p}=0.001$ & $t(14)=-3.68$ & $\mathrm{p}=0.002$ & $t(14)=-3.03$ & $\mathrm{p}=0.008$ & $t(14)=-1.43$ & $\mathrm{p}=0.17$ \\
\hline 14 DPI & $t(13)=-1.41$ & $\mathrm{p}=0.18$ & $t(13)=0.13$ & $\mathrm{p}=0.90$ & $t(13)=0.95$ & $\mathrm{p}=0.36$ & $t(13)=0.54$ & $p=0.02$ \\
\hline $30 \mathrm{DPI}$ & $t(13)=-3.81$ & $\mathrm{p}=0.002$ & $\mathrm{U}(14)=11$ & $\mathrm{p}=0.03$ & $\mathrm{U}(13)=21$ & $p=0.46$ & $t(14)=2.56$ & $p=0.59$ \\
\hline 90 DPI & $t(14)=2.83$ & $\mathrm{p}=0.1$ & $t(14)=-6.63$ & $\mathrm{p}<0.001$ & $t(14)=-2.20$ & $\mathrm{p}=0.04$ & $t(13)=-2.89$ & $\mathrm{p}=0.01$ \\
\hline \multirow[t]{2}{*}{150 DPI } & $t(18)=-4.51$ & $\mathrm{p}=0.002$ & $t(18)=4.52$ & $\mathrm{p}<0.001$ & $t(18)=5.12$ & $\mathrm{p}<0.001$ & $t(18)=2.40$ & $p=0.03$ \\
\hline & NOT & $p$ & VC & $p$ & SNpr & $p$ & $\mathrm{CPu}$ & $p$ \\
\hline 7 DPI & $t(14)=-2.94$ & $\mathrm{p}=0.01$ & $t(14)=0.76$ & $\mathrm{p}=0.47$ & $t(14)=-0.69$ & $\mathrm{p}=0.50$ & $t(14)=-1.24$ & $\mathrm{p}=0.23$ \\
\hline 14 DPI & $t(13)=-0.46$ & $p=0.66$ & $t(13)=-0.74$ & $\mathrm{p}=0.47$ & $t(13)=0.43$ & $p=0.67$ & $t(13)=0.34$ & $\mathrm{p}=0.74$ \\
\hline $30 \mathrm{DPI}$ & $t(14)=2.56$ & $\mathrm{p}=0.02$ & $t(13)=-2.28$ & $\mathrm{p}=0.04$ & $t(14)=1.25$ & $p=0.23$ & $t(14)=0.98$ & $\mathrm{p}=0.34$ \\
\hline 90 DPI & $t(14)=-1.94$ & $\mathrm{p}=0.07$ & $t(12)=-0.82$ & $\mathrm{p}=0.42$ & $t(13)=-3.12$ & $\mathrm{p}=0.008$ & $t(13)=-2.85$ & $\mathrm{p}=0.01$ \\
\hline 150 DPI & $t(18)=1.27$ & $\mathrm{p}=0.22$ & $t(18)=2.32$ & $\mathrm{p}=0.03$ & $t(18)=2.16$ & $\mathrm{p}=0.04$ & $t(18)=2.99$ & $p=0.007$ \\
\hline
\end{tabular}

Table 3. Test statistics for histological GFAP data. $t$ indicates a student's $t$ test; $U$ indicates that data failed normality and a Mann Whitney Rank-Sum analysis was performed. Abbreviations not included with Table 2: SCN: Suprachiasmatic Nucleus, VC: Visual Cortex; SNpr: Substantia Nirgra pars reticulate; Cpu: Caudoputamen.

In the dLGN, there was no significant difference in GFAP expression in astrocytes between injured and sham mice 7, 14, or 90 DPI (sup. fig. 4b,e; table 3). There was increased astrogliosis in TBI mice in the dLGN 30 and 150 DPI. The pattern of astrogliosis is the same in the vLGN with no significant effects of TBI in mice 7, 14, or 90 DPI, while there are delayed increases 30 DPI and chronic resurgence 150 DPI (sup. fig. 6b, e). There are no statistically significant changes to microglia at any time in the dLGN (table 4); however, a few amoeboid microglia can be found at various time points (see sup. fig. 4c, f). The vLGN shows a similar pattern of microgliosis as it does for astrogliosis (tables 3 \&4respectively) with delayed worsening in the TBI group until 30 and 90 DPI, with subsequent declines in microglial activation. Seven DPI and 14 DPI there were no significant differences between sham and TBI, but, again, there are a few amoeboid microglia present by 14 DPI (sup. fig. 6c, f). 
Suprachiasmatic Nucleus. We previously showed no changes to the SCN in our TBI model 7 or 30 DPI $[12,14]$ but at later time points we see differences arise in this direct hypothalamic target of the optic nerve. There was no degeneration visible at any time point, - somatic nor axonal (sup. fig. 8a, table 2). GFAP expression was significantly higher in TBI animals at our latest time point 150 DPI (table 3). GFAP staining intensity was not different at any other time point (sup. fig. 8b,d). Perhaps even more intriguing, microglial soma sizes significantly increased in TBI mice in the SCN 14 DPI right between previous time points examined and long before astroglial responses (table 4). No significant microglial activation was observed in TBI mice 7, 30, 90, or 150 DPI (sup. fig. 8c, e). IBA-1 perimeter analyses resulted in the same significance or insignificance as their respective area analyses (sup. table 1).

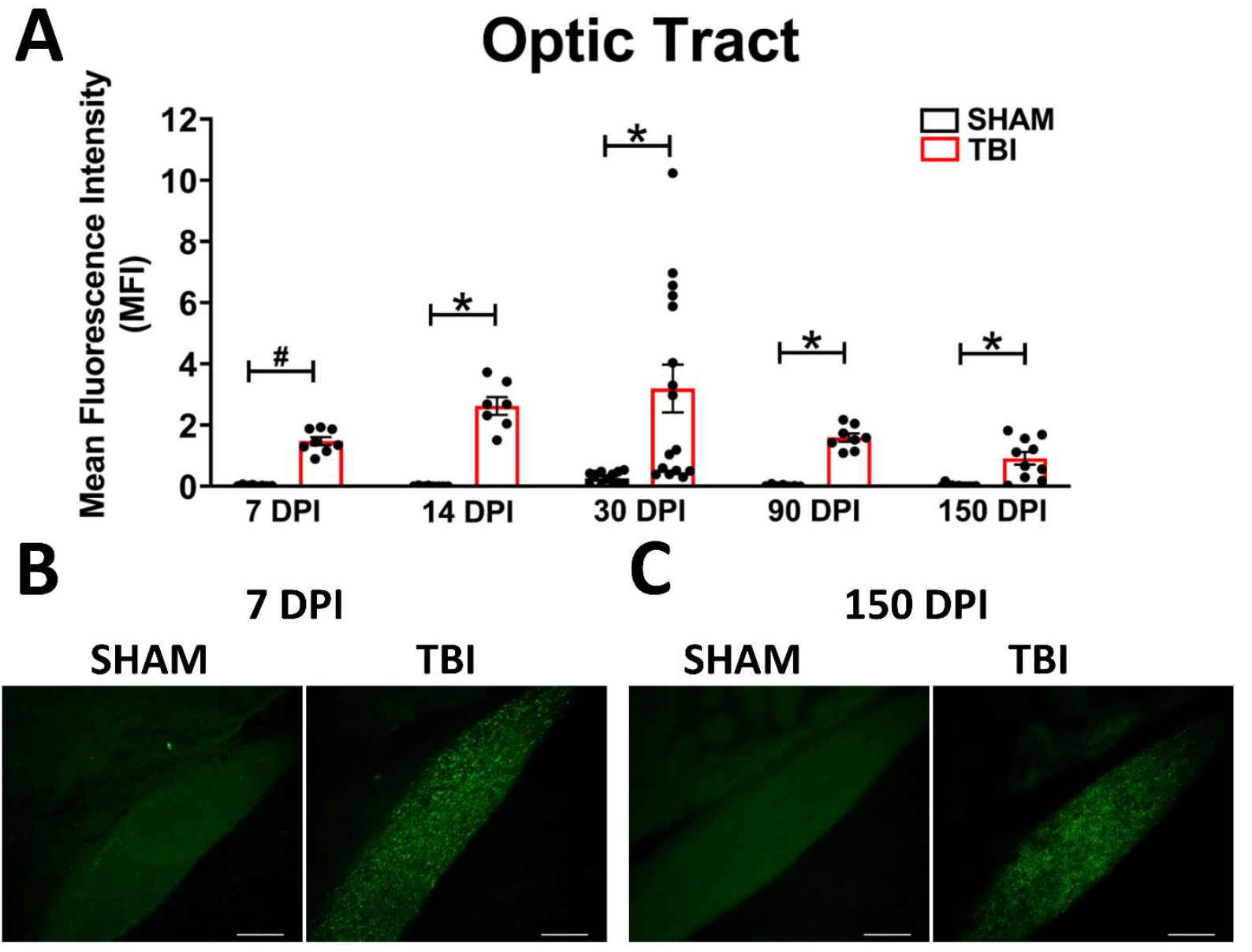

Figure 2. Degeneration in this model begins in the optic nerve and propagates through the optic tract throughout the time course. (A) There is significant degeneration (FJ-C staining) in injured mice at all time points compared to sham. Representative images of degeneration early (B) and chronically (C) after TBI taken at 20x magnification with a scale bar representing $100 \mu \mathrm{m} .{ }^{*} \mathrm{p}<0.001$, \# $\mathrm{p}<0.05$ for sham vs TBI within a time point.

Superior Colliculi. Degeneration is limited to the superficial input layers of the superior colliculi with no positive FJ-C staining in the deep output layers. Degeneration was significantly higher in TBI mice at all time-points (table 2). See supplementary figure 9a and $9 \mathrm{~d}$ for data and selected images, and supplementary figure $3 \mathrm{~g}$ for subdivisions of the SC. Interestingly, only 30 DPI FJ staining intensity was bimodal (low v. high $p<0.001$; low v. sham $p=0.06$; high v. sham $\mathrm{p}=0.001$; sup. fig. 10). As in the OT, there is significantly increased astrogliosis in TBI mice 7 DPI (table 3). However, while the OT showed possible improvement in astrogliosis $30 \mathrm{DPI}$, this improvement arises 14 DPI in the superior colliculi and returns for all following time points (sup. fig. 9b, e). Inflammatory microglial states were only significantly present in TBI superior colliculi 150 DPI (table 4), but in looking at the images taken at each time point there were at least a few amoeboid IBA-1 positive cells at all time points and there is a clear difference in microglial morphology more indicative of the activating states (sup. fig. 9c, f). Soma area measured 7, 30, 90 and 150 DPI showed no increased microglial soma size, and these results were consistent with perimeter changes (sup. table 1).

Accessory Optic Tract Nuclei. We found punctate FJ-C staining in both dorsal and medial terminal nuclei. Degeneration was significantly increased in the DTN at all time points (sup. fig. 11a, d; table 2) and high vs low expression was bi-modal and significantly different at 30 DPI ( $p=0.002$; low $v$ sham $p=0.04$, high $v$ sham $=p<0.001$; sup fig. 12). The MTN also showed increased FJ-C at all time points except 150 DPI (sup. fig. 13a, d). A bi-modal distribution arose at 7 
(low $\mathrm{v}$. high $\mathrm{p}=0.008$, low $\mathrm{v}$. sham $\mathrm{p}=0.06$, high $\mathrm{v}$. sham $=\mathrm{p}<0.001$ ) and 30 DPI (low $\mathrm{v}$. high $\mathrm{p}=0.03$, low $\mathrm{v}$. sham $\mathrm{p}=0.004$, high v. sham $=p=0.004$; sup. fig. 14). Similar to the superior colliculi rather than the optic tract, the dorsal terminal nucleus of TBI mice is significantly more astrogliotic than sham at all times except 14 DPI (sup. fig. 11b, e; table 3). The MTN, on the other hand, shows possible recovery both 14 and 30 DPI with early increases in activation 7 DPI and later return 90 and 150 DPI (sup. fig. 13b, e). Inflammatory microglial morphology in the DTN shows persistently amoeboid structure at all time points (sup. fig. 11c, f; table 4). The MTN, however, never shows significant differences between groups (sup. fig. 13c, f).

\section{Table 4. Statistics for IHC - IBA-1 (Soma Area)}

\begin{tabular}{|c|c|c|c|c|c|c|c|c|c|c|}
\hline Time Point & OT & $p$ & dLGN & $p$ & vLGN & $p$ & SCN & $p$ & SC & $p$ \\
\hline 7 DPI & $t(13)=-3.4$ & $\# p=0.005$ & $\mathrm{U}(13)=22$ & $\mathrm{p}=0.33$ & $t(14)=-0.86$ & $\mathrm{p}=0.486$ & $t(14)=-0.16$ & $\mathrm{p}=0.87$ & $t(13)=0.62$ & $\mathrm{p}=0.55$ \\
\hline 14 DPI & $t(12)=-2.69$ & $\# \mathrm{p}=0.02$ & $t(13)=-0.78$ & $\mathrm{p}=0.45$ & $t(12)=-0.89$ & $\mathrm{p}=0.39$ & $t(13)=2.89$ & $\# \mathrm{p}=0.01$ & $t(13)=0.01$ & $\mathrm{p}=0.90$ \\
\hline 30 DPI & $t(11)=-3.04$ & $\# p=0.01$ & $t(14)=-0.58$ & $\mathrm{p}=0.58$ & $t(14)=-2.42$ & $\# \mathrm{p}=0.03$ & $t(13)=-0.08$ & $\mathrm{p}=0.94$ & $t(13)=-1.29$ & $\mathrm{p}=0.18$ \\
\hline 90 DPI & $t(14)=-4.19$ & ${ }^{*} \mathrm{p}<0.001$ & $t(14)=-0.40$ & $\mathrm{p}=0.57$ & $\mathrm{t}(14)=-2.50$ & $\# p=0.03$ & $t(13)=0.78$ & $\mathrm{p}=0.45$ & $t(14)=-0.8$ & $\mathrm{p}=0.44$ \\
\hline \multirow[t]{2}{*}{150 DPI } & $t(18)=-3.45$ & $\# p=0.003$ & $t(18)=-0.44$ & $\mathrm{p}=0.70$ & $t(18)=1.45$ & $\mathrm{p}=0.16$ & $t(18)=-0.33$ & $\mathrm{p}=0.74$ & $t(15)=-3.78$ & $\# \mathrm{p}=0.002$ \\
\hline & DTN & $p$ & MTN & $p$ & EW & $p$ & AON & $p$ & BoSC & $p$ \\
\hline 7 DPI & $t(13)=-3.26$ & $\# p=0.006$ & $t(13)=-0.68$ & $\mathrm{p}=0.51$ & $\mathrm{U}(13)=19$ & $\mathrm{p}=0.34$ & $t(13)=-1.62$ & $\mathrm{p}=0.13$ & $t(14)=-1.65$ & $\mathrm{p}=0.12$ \\
\hline 14 DPI & $t(13)=-4.99$ & ${ }^{*} \mathrm{p}<0.001$ & $t(13)=1.26$ & $\mathrm{p}=0.23$ & $t(13)=0.03$ & $\mathrm{p}=0.97$ & $t(13)=1.23$ & $\mathrm{p}=0.24$ & $t(13)=-2.4$ & $\# \mathrm{p}=0.03$ \\
\hline 30 DPI & $t(13)=7.79$ & ${ }^{*} \mathrm{p}<0.001$ & $t(11)=-1.85$ & $\mathrm{p}=0.09$ & $t(10)=-0.21$ & $\mathrm{p}=0.84$ & $\mathrm{~T}(13)=-2.09$ & $\mathrm{p}=0.06$ & $t(22)=1.80$ & $\mathrm{p}=0.08$ \\
\hline 90 DPI & $t(13)=-3.26$ & $\# p=0.006$ & $t(13)=-0.68$ & $\mathrm{p}=0.51$ & $\mathrm{U}(13)=19$ & $\mathrm{p}=0.34$ & $t(13)=-1.62$ & $\mathrm{p}=0.13$ & $t(8)=-1.76$ & $p=0.06$ \\
\hline \multirow[t]{2}{*}{150 DPI } & $\mathrm{U}(18)=16$ & $\# \mathrm{p}=0.01$ & $\mathrm{U}(18)=37$ & $\mathrm{p}=0.35$ & $t(16)=1.32$ & $\mathrm{p}=0.20$ & $t(18)=-0.59$ & $\mathrm{p}=0.57$ & $t(17)=3.1$ & $\# \mathrm{p}=0.006$ \\
\hline & NOT & $p$ & VC & $p$ & SNpr & $p$ & $\mathrm{CPu}$ & $p$ & & \\
\hline 7 DPI & $t(14)=-1.33$ & $\mathrm{p}=0.21$ & $t(14)=-0.26$ & $\mathrm{p}=0.80$ & $\mathrm{U}(14)=22$ & $\mathrm{p}=0.33$ & $t(13)=-2.44$ & $\# p=0.03$ & & \\
\hline 14 DPI & $t(13)=-1.19$ & $\mathrm{p}=0.47$ & $t(13)=-0.96$ & $\mathrm{p}=0.35$ & $t(13)=-0.32$ & $\mathrm{p}=0.75$ & $t(13)=-0.21$ & $\mathrm{p}=0.83$ & & \\
\hline 30 DPI & $t(18)=-0.49$ & $\mathrm{p}=0.63$ & $\mathrm{U}=42$ & $\mathrm{p}=0.26$ & $t(12)=-0.26$ & $\mathrm{p}=0.79$ & $t(14)=-1.03$ & $\mathrm{p}=0.32$ & & \\
\hline 90 DPI & $t(7)=0.81$ & $\mathrm{p}=0.36$ & $t(14)=-0.48$ & $p=0.26$ & $\mathrm{U}(14)=22$ & $\mathrm{p}=0.33$ & $t(13)=-2.44$ & $\# \mathrm{p}=0.03$ & & \\
\hline 150 DPI & $\mathrm{t}(17)=0.23$ & $\mathrm{p}=0.82$ & $\mathrm{t}(18)=0.07$ & $\mathrm{p}=0.65$ & $t(18)=-1.38$ & $\mathrm{p}=0.18$ & $t(17)=0.37$ & $\mathrm{p}=0.72$ & & \\
\hline
\end{tabular}

Table 4. Test statistics for histological IBA-1 soma area analyses. $t$ indicates a student's $t$ test; $U$ indicates that data failed normality and a Mann Whitney Rank-Sum analysis was performed. ${ }^{*} \mathrm{p}<0.001, \# \mathrm{p}<0.05$. See previous tables for abbreviations.

Brainstem Optic Nuclei. Nuclei involved in pupillary control and eye muscle coordination also receive input from RGCs; thus, it was also important to examine these regions despite previous literature finding no differences in the accessory optomotor nucleus[8]. Specifically, we examined the Edinger-Westphal Nuclei and Accessory Optomotor Nuclei, but there was no degeneration at any time-point in either region (sup. fig. 15d). We also saw no astroglial response in these regions with little to no positive GFAP staining present in either sham or TBI tissue; thus, we could not analyze GFAP intensity in these regions. There were also very few microglia present in both the Edinger-Westphal and accessory optic nuclei, which resulted in a low $\mathrm{n}$ for some of our analyses. Nonetheless, soma area and perimeter analyses revealed no significant differences at any time in either region (sup. fig. 15a-c).

Brachium of the Superior Colliculi. Degeneration was evident in this region 7, 14, 90, and 150 DPI. Thirty DPI there was no longer a significant difference between sham and TBI mice, and most mice showed no positive staining while others presented with a few bright punctate areas (sup. fig. 16a, b; table 2). Unexpectedly, astrogliosis in this region followed an almost opposite pattern with significant differences in TBI mice at only 30 and 150 DPI and no significant differences 7, 14, or 90 DPI (sup. fig. 17a, b; table 3). IBA-1 soma increases also arose in a different pattern with significant differences at 14 and 150 DPI (sup. fig. 18a, b; table 4). It is possible that microglial activation differences 
30 DPI were obscured as this white matter tract is thin in coronal sections and circular amoeboid characteristics appear more elongated supporting our significant finding in soma perimeter but not area $30 \mathrm{DPI}$ (sup. table 1). There were no differences in IBA-1 soma area 7, 30, or 90 DPI.

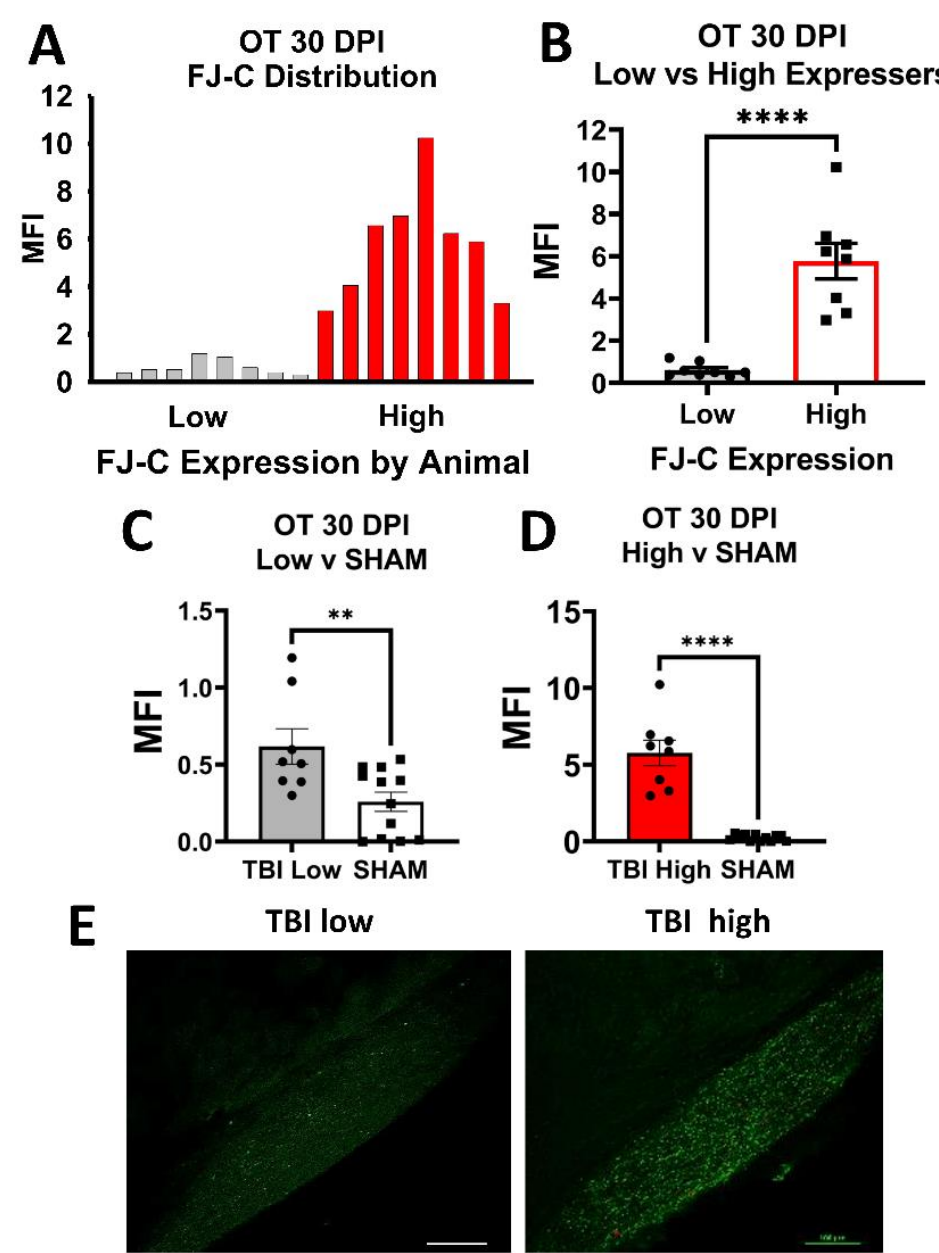

Figure 3. FJ-C expression showed distinct bi-modal characteristics in the OT at 30 DPI. (A) We plotted a frequency distribution of TBI mice and saw two clear distributions of "low" and "high" FJ-C expression. (B) These two groups were significantly different from each other potentially indicating inherent differences in recovery from, or susceptibility to, injury in the model of optic axonal injury. (C) Low FJ in TBI mice, however, was still significantly high than sham-injured mice (D) as were high FJ-C expressing mice. (E) We can clearly see little to no degeneration in the low group compared to the high with these representative photomicrographs taken at 20x magnification. Scale bars represent 100um. MFI: Mean Fluorescence, OT: Optic Tract, Intensity, ${ }^{* *} \mathrm{p}<0.01,{ }^{* * *} \mathrm{p}<0.001,{ }^{* * * *} \mathrm{p}<0.0001$.
Nucleus of the Optic Tract. Unlike most regions examined, positive FJ-C staining does not arise in the nucleus of the optic tract until two weeks after injury, after which it remains significantly worse than sham at all time-points (sup. fig. 16a, c; table 2). In the NOT, there was a significant increase in GFAP fluorescence intensity in astrocytes in TBI mice 7 or 30 DPI (table 3). There were no significant increases 14,90 , or 150 DPI (sup. fig. 17a, c). Microglial activation in TBI mice 7, 14, 30,90 , and 150 DPI was also not significant (sup. fig. 18a, c; table 4).

Visual Cortex. There was no positive FJ-C staining in the visual cortex (sup. fig. 19a), but there is a delayed glial response. Increased GFAP intensity in TBI mice was present at only 30 DPI and 150 DPI but not 7 DPI, 14 DPI, or 90 DPI (sup. fig. b, d; table 3). This is particularly interesting for three reasons. First, 30 DPI is a time point when astrogliosis in the OT is alleviated. Second, there is another split in TBI mice 30 DPI with very little spread between high vs. low groups, while at 150 DPI all animals have increased astrogliosis compared to shams. Third, positive GFAP staining 30 DPI is not only more intense, but also shows very different morphological profiles of the astroglia that we do not see at any other time point including 150 DPI. Finally, while there were no significant differences in IBA-1 soma area between sham and TBI mice in this time course (sup. fig. 19c, e; table 4), soma perimeter was significantly increased in TBI mice 30 DPI (sup. table 1). As was seen in the SC, there is a discernable difference between sham and TBI microglial morphology in terms of their processes (sup. fig. 19c).

The Visual Corticostriatal Loop. Higher order processing and motor coordination of vision also involve the caudoputamen and substantia nigra pars reticulata which receive projections from and project to the SC. While there was no positive FJ-C staining at any time point in the caudoputamen (sup. fig. 20a), the tail of the caudoputamen displayed delayed increases in astrogliosis 90 and 150 DPI with no significant differences at acute or subacute time points 7,14 , or $30 \mathrm{DPI}$

(sup. fig. $20 \mathrm{~b}, \mathrm{~d}$ ). There is also both early (7 DPI) and delayed (90 DPI) microglial response with no differences sub acutely (14 or 30 DPI) or chronically (150 DPI; sup. fig. 20 c, e). There was also no degeneration in the SNR (sup. fig. 21a), but there was increased astrogliosis 90 and 150 DPI (sup. fig. 21b, d) possibly indicating that glial cells are communicating in a slower manner, but in the absence of neuronal cell death requiring long periods of time following TBI. There is, however, no microglial response in this region (sup. fig. 21c, e). See table 3 for GFAP and table 4 for IBA-1 statistical reports. 


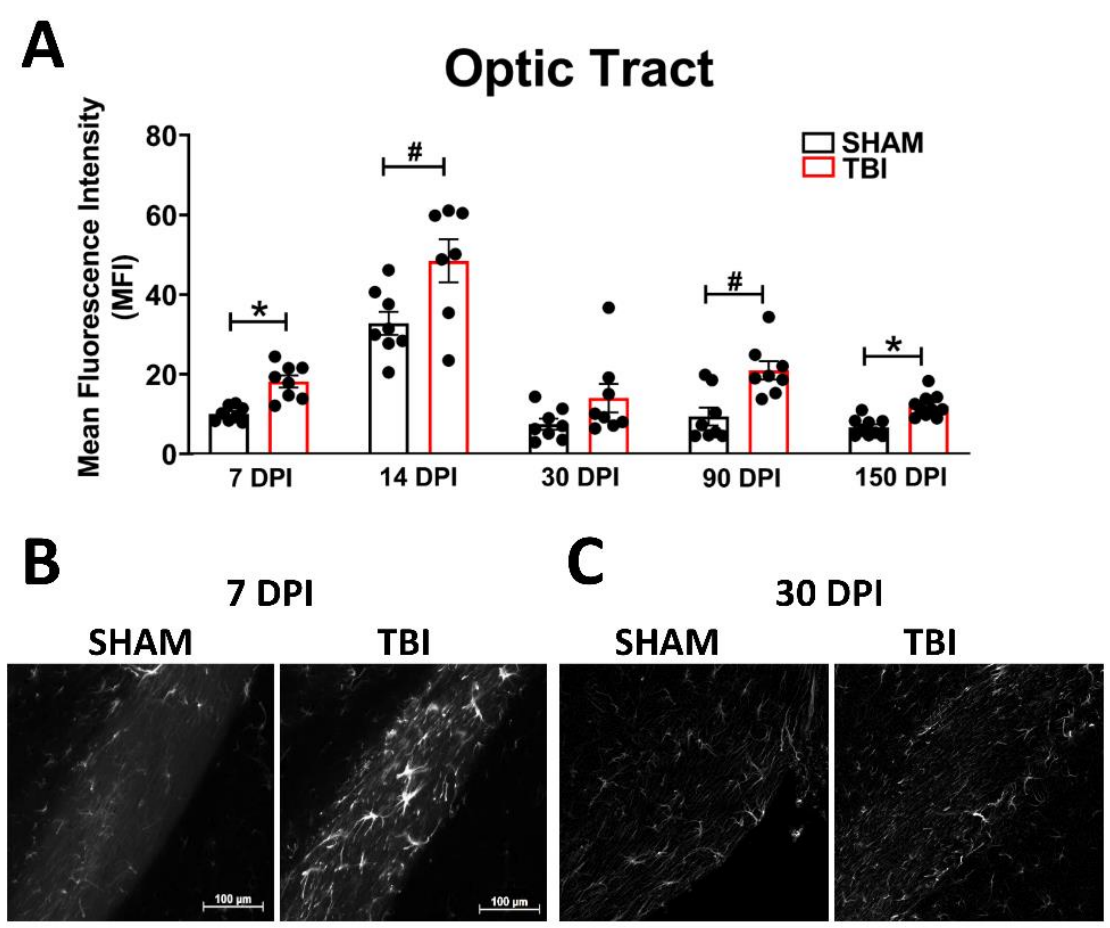

Figure 4. Astrogliosis in the optic tract is marked by early activation, recovery, and subsequent re-activation after TBI. GFAP immunofluorescence is significantly increased in the optic tract at all time points except 30 DPI (A). (B) Representative images of TBI vs sham mice 7 DPI reveal not only brighter GFAP but also more astrocytes (not quantified) compared to similar levels of both 30 DPI (C). Representative photomicrographs were taken at 20x magnification with a scale bar representing $100 \mu \mathrm{m} .{ }^{*} \mathrm{p}<0.001, \# \mathrm{p}<0.05$ for sham vs TBI within a time point.

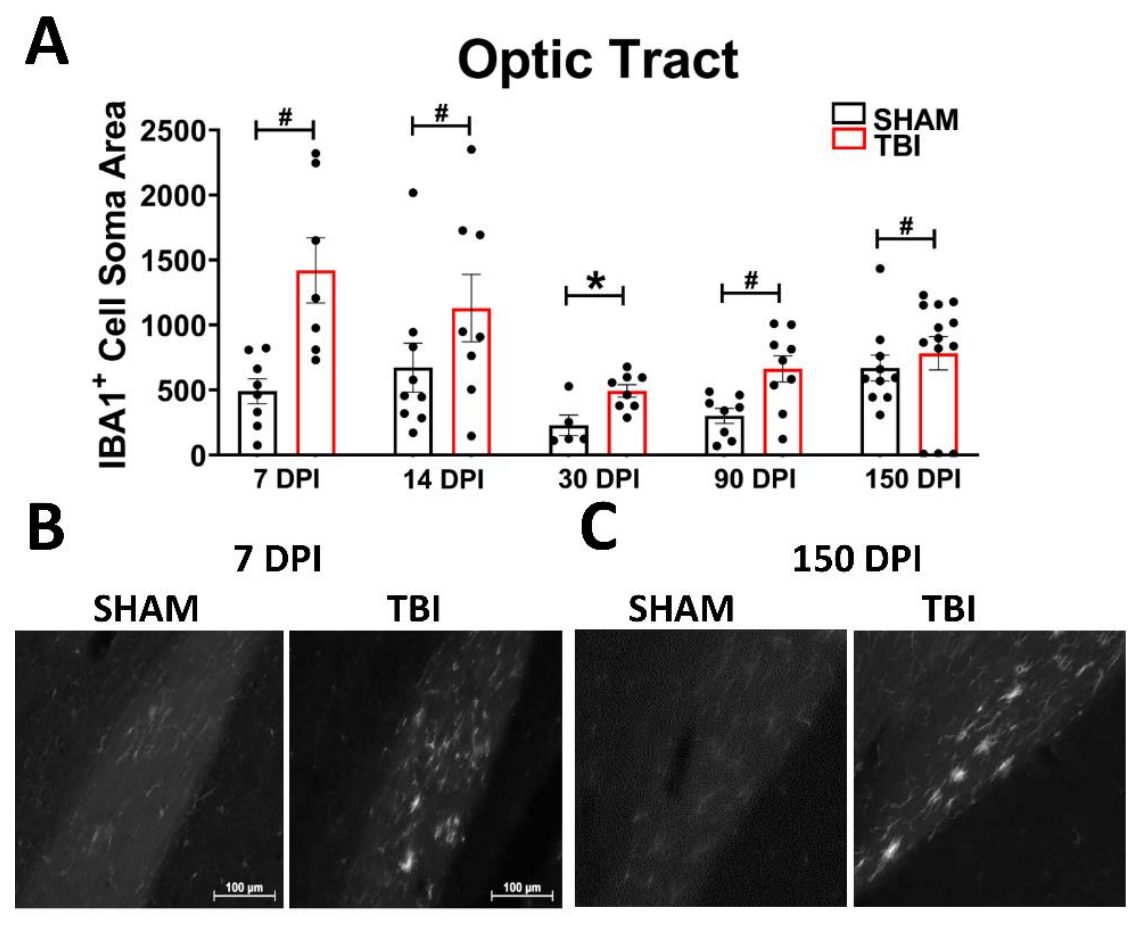

Figure 5. Microglial morphology is persistent in the optic tract. As ameboid microglia represent an immune response and phagocytosis of debris (in this case axonal), we examined soma size of IBA-1 positive cells in the OT at all time points (A). The spherical, bright, ameboid cells can be seen at 7 DPI (B) and 150 DPI (C). Representative photomicrographs were taken at 20x magnification with a scale bar representing $100 \mu \mathrm{m} .{ }^{*} \mathrm{p}<0.001, \# \mathrm{p}<0.05$ for sham vs TBI within a time point. 


\section{Discussion}

In the current study, we show that the adolescent murine visual system is chronically and dynamically vulnerable to a single, mild blunt head injury. Following blunt head trauma, adolescent male mice present with chronic axonal degeneration (visible through punctate FJ-C staining) in the optic nerve to lateral geniculate nuclei, superior colliculi, and into the accessory optic system. We show the potential presence of multi-synaptic signaling from axonal degeneration through the brachium of the superior colliculus to the nucleus of the optic tract despite absence of neuronal death. Additionally, we show evidence for a critical period between 14-30 days after injury when the visual system may be recovering/remodeling. This is supported by variable FJ-C accumulation within the TBI group starting 30 DPI and, in some regions, continuing through the duration of the time-course.

We also show chronic differences in gliosis patterns 14-150 DPI that to our knowledge have not yet been reported. While not as consistent across the five time points examined as degeneration, gliotic response to injury appears to be more pervasive in that changes arise at some points along our time course in every region examined (save the brainstem). This may indicate that glia are able to respond to optic nerve injury even in regions with no detectable degeneration. Moreover, this multi-synaptic glial communication could mean that degeneration may arise in cortical/secondary regions given a longer period of time after TBI. Our IBA-1 data also suggest that neuroinflammation, in at least some cases, is a slow, potentially progressive process that can arise as late as 150 days after injury (e.g., the superior colliculi and visual cortex).

Many of our findings are in line with the slow Wallerian degeneration (WD) that occurs after axonal injury in the central nervous system (CNS). WD progresses through mechanisms separate from apoptotic cell death, [20] can occur without causing neuronal loss, [39] and usually does not allow regeneration of lost axons/neurons in the CNS. If repair is possible under WD mechanisms/processes, it typically requires months to years following axotomy or TAI [17,40]. In a longitudinal study for 13 weeks (84 days) after optic nerve stretch, authors showed persistent die back of intact nerve fibers in the optic tract, as well as continually decreasing myelination and evident Wallerian pathologies [41]. Our data are consistent with this possibility, and we suggest that, later after injury, chronic Wallerian degeneration may begin to slow (such as in regions like the medial terminal nuclei and LGN where Fluoro-jade staining is no longer significantly present $150 \mathrm{DPI})$. It is also possible that degenerating nerve fibers may be able to initiate additional degeneration long after initial injury and axon dieback.

TAI studies exploring the causes of slow WD suggest that residual myelin debris and incomplete clearance of digestion chambers containing dead axon segments could be the driving factor for chronic WD and persistent/ongoing degeneration. Our FJ staining with its punctate characteristics throughout the optic tract and projection targets, which persists through 150 DPI may be labeling this residual myelin debris, supporting the notion of prolonged lack of debris clearance. Additionally, support for incomplete debris removal could come from our glial data. Both astrocytes and microglia can perform phagocytic clearance of cellular/axonal debris [42-44], and this ability, at least in microglia, can become dysregulated after experimental TBI [45]. The lack of amoeboid/phagocytic microglia in regions high in FJ-C staining, like the dLGN and MTN, and their delayed morphological change in most other regions outside the OT could indicate that glia fail to clear the debris up to 150 DPI in mice. Direct measures of microglial activity and function, however, are needed to confirm this idea.

Further, the axonal degeneration seen in grey matter and secondary regions (i.e., regions not directly connected to the OT) along with delayed glial responses indicates potential trans-synaptic WD following TBI in regions like the VC. However, we should note that we did not see degeneration in the single-synaptic region of the suprachiasmatic nuclei, nor in the trans-synaptic deeper brainstem projection nuclei (EW/AON) or cortical regions (VC). While this lack of cortical and SCN degeneration is consistent with our previously published results [12,14], it is interesting that brainstem projections are also unaffected by this injury. The Edinger-Westphal nucleus is a compelling region for examination after optic nerve injury due to its proximity to the oculomotor complex (aka, AON, which is also unaffected) and the fact that damage to the retina and optic nerve is often reflected in loss of proper pupillary function [46]. However, the connection between optic nerve and EW is multi-synaptic. Moreover, we proposed in our previous work that lack of SCN response to injury may be because optic nerve projections here are made up primarily of axons from the more resilient intrinsically photosensitive retinal ganglion cells (ipRGCs). ipRGCs respond to light cues in the retina, and project primarily to SCN. They also project to the pretectal nuclei, which signal to the EW for pupillary dilation [47].

It also seems that roughly one month after injury, mice appear to split into at least two groups: one with high FJC staining and one with levels near sham mice. These splits are seen 30 DPI in the optic tract, LGN, SC, and AOS; 90 and 150 DPI in the LGN (potentially mirrored in glial response); and as early as 7 DPI in the MTN. Despite continued degeneration in the OT at 30 DPI, the significant reduction in a subset of mice at 30 DPI in almost all direct projection regions may indicate that $30 \mathrm{DPI}$ is the critical time at which a decision is made to protect the brain from further effects of injury or not. This is particularly interesting when considering the fact that we saw low FJ staining in projection 
regions 90 and 150 DPI while the OT remained high, indicating that the OT at 30 DPI may dictate how the downstream targets respond over time. It is important to note that our 30 DPI time point had a higher number of mice than other times due to the addition of the replacement cohort. It may be that this increased number of subjects allowed more statistical power to detect a divergence between low-degeneration groups and high-degeneration groups. Thus, larger cohorts may be necessary to determine exactly when a subset of mice begins to show resiliency to injury. However, although the other 4 time points had lower n, we still saw bi-modal distributions in multiple regions at 7, 90, and/or 150 DPI indicating that the split distributions were likely not the result of two different cohorts. Additionally, the high/low groups at 30 included animals from both the original cohort and the added second cohort. We were unable to follow individual mice across time points in order to track individual differences due to the invasive and terminal nature of histologic measures. Future research is needed to determine whether this is due to individual differences or presence of an unknown factor promoting glial clearance of axonal debris.

WD also includes recovery and delayed progression of axonal degeneration, and involves multiple cell types in tissue surrounding injury [26] including both astrocytes and microglia [48,49]. Tissue responses to injury include the production of "astroglial scars," which can both promote regeneration [50-52] and prevent axon regrowth/repair [50]. Glial scars involve both astrocytic and microglial pro- and anti-inflammatory responses to injury [53-55], and glia direct CNS responses to injury and disease [49]. For example, microglia-astrocyte coordination is likely required for axon survival and regeneration where lack of a microglial response shifts astrocytes to a pro-inflammatory state in a mouse model of demyelination [56]. Yet, studies probing the activity of glial cells during regeneration after traumatic axonal injury, in particular, are lacking [22,57]. Our data suggest that astrocytes and microglia respond differently in relation to each other within regions at each time point, such as in the LGN where astrocytes are more consistently reactive compared to microglia, with no microglial response in the dLGN vs vLGN. This also appears true in secondary projection regions like the NOT where there is no microglial morphology change despite early increased GFAP expression and FJ-C staining, or in the BoSC where the critical/recovery period we see arises later than in the OT or LGN. This nonparallel response between glial cell types may explain why studies focusing on microglia or astrocytes alone have only been partially successful [58,59].

Our data argue not only for co-activation of glial cells in a region-dependent manner but also in a time-dependent manner. While temporal coordination/dependence of microglial and astrocyte activation is not well understood, one study showed that microglia are dysregulated shortly after brain injury with possible recovery in a time-dependent manner [45]. Although we did not directly examine microglial signaling pathways, our data are consistent with the possibility of reduced inflammatory response 14-30 DPI. We also support the notion of persistent glial dysfunction. For example, ameboid microglia and reactive astrocytes persist throughout the time-course in the optic tract and LGN, but degeneration remains. Moreover, in the SC, where microglial changes are not seen until 150 DPI, this could indicate a delayed spread of inflammatory signals, delayed response of microglia despite inflammatory signals, or even an impaired ability for microglia to sense the inflammation if it is present in these more distal regions. We also report subacute and chronic glial activation without degeneration in the visual cortex and corticostriatal loop as well as concordant appearance of both astrocytes and microglia in predominantly grey-matter regions like the LGN, SC, and VC potentially implicating signaling events we have yet to elucidate.

Not only did one glial type lack activation in some regions, but astrocytes and microglia also sometimes reversed which was significantly active (e.g., microglia are activated earlier than astrocytes in the caudoputamen and SCN, while astrocytes are reactive before microglia change in the SC). These differences may suggest that discovering how and when glial cells communicate after injury is critical to long-term treatment strategies after axonal injury. At longer time points, we may see microglia beginning to clear the axonal debris. Alternatively, because our 14 and 30 DPI critical periods of reduced glial activation and degeneration are a bit earlier than the 60 DPI time-point examined by Izzy, et al. [45], it is possible that the time-course of inflammatory response is slightly different after TAI compared to cortical injury. Future studies would need to follow these processes comparing the different types of injury.

\section{Conclusions}

Our data provide evidence for a window of time in which the optic/visual system may be recovering from blunt head injury, because TBI and sham mice do not differ at subacute time points (14-30 DPI), outside the OT. Furthermore, there appears to be a subset of mice that have significantly less degeneration after head trauma from 30-150 DPI, and given that there is not a stratification of severity prior to these times (by histologic means), this suggests there may be individuals with improved long-term recovery. We show that chronic visual system changes after head injury are characterized by tissue responses in the brain, in addition to previously reported retinal thinning and RGC loss. These tissue responses include progressive degeneration and/or gliosis in multiple direct and multi-synaptic cortical and thalamic (but not brainstem) optic nerve projection targets, which worsen chronically after an initial period of apparent recovery. 
Degeneration, however, is not necessarily predictive of gliotic responses. Rather, its chronic presence may simply be the trigger for occasionally delayed inflammatory. This was shown through dynamic glial response to injury that was dependent upon projection region and time after said injury. Overall, these data support a potential timeline for interventions after TBI/TAI in the visual system beyond the acute post-injury phase.

\begin{tabular}{|c|c|c|c|}
\hline Traumatic Brain Injury & TBI & Standard deviation & SD \\
\hline traumatic axonal injury & TAI & Optic Tract & OT \\
\hline indirect traumatic optic neuropathy & iTON & dorsal lateral geniculate nucleus & dLGN \\
\hline Wallerian Degeneration & WD & Ventral lateral geniculate nucleus & vLGN \\
\hline oxygen & $\mathrm{O}_{2}$ & suprachiasmatic nucleus & $\mathrm{SCN}$ \\
\hline Days post injury & DPI & superior colliculi & SC \\
\hline immunohistochemical & IHC & Dorsal terminal nucleus & DTN \\
\hline phosphate-buffered saline & PBS & medial terminal nucleus & MTN \\
\hline Fluoro-Jade C & FJ-C & accessory optomotor nucleus & $\mathrm{AON}$ \\
\hline glial fibrillary acidic protein & GFAP & Edinger-Westphal nuclei & EW \\
\hline ionized calcium-binding adaptor molecule 1 & Iba-1 & brachium of the superior colliculus & BoSC \\
\hline Analysis of variance & ANOVA & nucleus of the optic tract & NOT \\
\hline Standard error of the mean & SEM & visual cortex & $\mathrm{VC}$ \\
\hline Region of interest & ROI & Central nervous system & CNS \\
\hline Mean & M & & \\
\hline
\end{tabular}

Table 5. Abbreviations in order of appearance in the text.

\section{Supplementary Materials:}

Author Contributions: Conceptualization, Shelby M. Cansler and Nathan K. Evanson; Formal analysis, Shelby M. Cansler, Emily M. Shalosky, and Jordyn N. Torrens; Funding acquisition, Nathan K. Evanson; Investigation, Shelby M. Cansler, Emily M Shalosky, Jordyn N. Torrens, and Nathan K. Evanson; Methodology, Shelby M. Cansler, Emily M. Shalosky, Jordyn N. Torrens, and Nathan K. Evanson; Supervision, Nathan K. Evanson; Visualization, Shelby M. Cansler, Emily M. Shalosky, and Jordyn N. Torrens; Writing - original draft, Shelby M. Cansler, Emily M. Shalosky, Jordyn N. Torrens, and Nathan K. Evanson; Writing - review \& editing, Shelby M. Cansler, Emily M. Shalosky, Jordyn N. Torrens, and Nathan K. Evanson..

Funding: This work was supported by the National Institutes of Health [NIH grant HD001097 (NKE)], and by a Procter Award and the Division of Pediatric Rehabilitation Medicine at Cincinnati Children's Hospital Medical Center (NKE). Funding entities played no role in the design, execution, analysis, or interpretation of results.

Acknowledgments: We thank Jordyn Torrens and Emily Shalosky for technical assistance in performing the studies described. 
Conflicts of Interest: The authors declare no conflict of interest.

\section{References}

1. E A Finkelstein; P S Corso, T.R.M. Incidence and economic burden of injuries in the United States. J Epidemiol Community Health 2007, 61, 926-926, doi:10.1136/jech.2007.059717.

2. Selassie, A.W.; Zaloshnja, E.; Langlois, J.A.; Miller, T.; Jones, P.; Steiner, C. Incidence of Long-term Disability Following Traumatic Brain Injury Hospitalization, United States, 2003. The Journal of Head Trauma Rehabilitation 2008, 23, 123-131, doi:10.1097/01.Htr.0000314531.30401.39.

3. Zaloshnja, E.; Miller, T.; Langlois, J.A.; Selassie, A.W. Prevalence of long-term disability from traumatic brain injury in the civilian population of the United States, 2005. J Head Trauma Rehabil 2008, 23, 394-400, doi:10.1097/01.HTR.0000341435.52004.ac.

4. Chen, C.; Peng, J.; Sribnick, E.A.; Zhu, M.; Xiang, H. Trend of Age-Adjusted Rates of Pediatric Traumatic Brain Injury in U.S. Emergency Departments from 2006 to 2013. Int J Environ Res Public Health 2018, 15, 1171, doi:10.3390/ijerph15061171.

5. Asma Bashir; A. Abebe, Z.; A. Mcinnes, K.; B. Button, E.; Igor Tatarnikov; Hang Cheng, W.; Margalit Haber; Anna Wilkinson; Carlos Barron; Ramon Diaz-Arrastia; et al. Increased severity of the CHIMERA model induces acute vascular injury, sub-acute deficits in memory recall, and chronic white matter gliosis. Experimental Neurology 2020, 324, 113116, doi:10.1016/j.expneurol.2019.113116.

6. $\quad$ Finnanger, T.G.; Olsen, A.; Skandsen, T.; Lydersen, S.; Vik, A.; Evensen, K.A.I.; Catroppa, C.; Håberg, A.K.; Andersson, S.; Indredavik, M.S. Life after Adolescent and Adult Moderate and Severe Traumatic Brain Injury: Self-Reported Executive, Emotional, and Behavioural Function 2-5 Years after Injury. Behavioural Neurology 2015, 2015, 329241, doi:10.1155/2015/329241.

7. Koliatsos, V.E.; Cernak, I.; Xu, L.; Song, Y.; Savonenko, A.; Crain, B.J.; Eberhart, C.G.; Frangakis, C.E.; Melnikova, T.; Kim, H.; et al. A Mouse Model of Blast Injury to Brain: Initial Pathological, Neuropathological, and Behavioral Characterization. Journal of Neuropathology \& Experimental Neurology 2011, 70, 399-416, doi:10.1097/NEN.0b013e3182189f06.

8. Keating, C.E.; Browne, K.D.; Duda, J.E.; Cullen, D.K. Neurons in Subcortical Oculomotor Regions are Vulnerable to Plasma Membrane Damage after Repetitive Diffuse Traumatic Brain Injury in Swine. Journal of Neurotrauma 2020, doi:10.1089/neu.2019.6738.

9. Gennarelli, T.A.; Thibault, L.E.; Tipperman, R.; Tomei, G.; Sergot, R.; Brown, M.; Maxwell, W.L.; Graham, D.I.; Adams, J.H.; Irvine, A.; et al. Axonal injury in the optic nerve: a model simulating diffuse axonal injury in the brain. Journal of Neurosurgery 1989, 71, 244, doi:10.3171/jns.1989.71.2.0244.

10. Guley, N.H.; Rogers, J.T.; Del Mar, N.A.; Deng, Y.; Islam, R.M.; D'Surney, L.; Ferrell, J.; Deng, B.; Hines-Beard, J.; Bu, W.; et al. A Novel Closed-Head Model of Mild Traumatic Brain Injury Using Focal Primary Overpressure Blast to the Cranium in Mice. J Neurotrauma 2016, 33, 403-422, doi:10.1089/neu.2015.3886.

11. Yu-Wai-Man, P. Traumatic optic neuropathy-Clinical features and management issues. Taiwan journal of ophthalmology 2015, 5, 3-8, doi:10.1016/j.tjo.2015.01.003.

12. Evanson, N.K.; Guilhaume-Correa, F.; Herman, J.P.; Goodman, M.D. Optic tract injury after closed head traumatic brain injury in mice: A model of indirect traumatic optic neuropathy. PLoS One 2018, 13, e0197346, doi:10.1371/journal.pone.0197346.

13. Guilhaume-Correa, F.; Cansler, S.M.; Shalosky, E.M.; Goodman, M.D.; Evanson, N.K. Greater neurodegeneration and behavioral deficits after single closed head traumatic brain injury in adolescent versus adult male mice. J Neurosci Res 2020, 98, 557-570, doi:10.1002/jnr.24535.

14. Cansler, S.M.; Guilhaume-Correa, F.; Day, D.; Bedolla, A.; Evanson, N.K. Indirect traumatic optic neuropathy after head trauma in adolescent male mice is associated with behavioral visual impairment, neurodegeneration, and elevated 
endoplasmic reticulum stress markers at acute and subacute times. bioRxiv 2020, 2020.2006.2011.144766, doi:10.1101/2020.06.11.144766.

15. Chan, J.W.; Hills, N.K.; Bakall, B.; Fernandez, B. Indirect Traumatic Optic Neuropathy in Mild Chronic Traumatic Brain Injury. Investigative Ophthalmology \& Visual Science 2019, 60, 2005-2011, doi:10.1167/iovs.18-26094.

16. Chan, J.; Tsui, E.; Peh, W.; Fong, D.; Fok, K.; Leung, K.; Yuen, M.; Fung, K. Diffuse axonal injury: detection of changes in anisotropy of water diffusion by diffusion-weighted imaging. Neuroradiology 2003, 45, 34-38.

17. Vargas, M.E.; Barres, B.A. Why Is Wallerian Degeneration in the CNS So Slow? Annual Review of Neuroscience 2007, 30, 153179, doi:10.1146/annurev.neuro.30.051606.094354.

18. Marion, C.M.; Radomski, K.L.; Cramer, N.P.; Galdzicki, Z.; Armstrong, R.C. Experimental Traumatic Brain Injury Identifies Distinct Early and Late Phase Axonal Conduction Deficits of White Matter Pathophysiology, and Reveals Intervening Recovery. J Neurosci 2018, 38, 8723-8736, doi:10.1523/jneurosci.0819-18.2018.

19. Conforti, L.; Gilley, J.; Coleman, M.P. Wallerian degeneration: an emerging axon death pathway linking injury and disease. Nature Reviews Neuroscience 2014, 15, 394-409, doi:10.1038/nrn3680.

20. Carroll, S.L.; Worley, S.H. Wallerian Degeneration is. In Reference Module in Neuroscience and Biobehavioral Psychology; Elsevier: 2017.

21. Arfanakis, K.; Haughton, V.M.; Carew, J.D.; Rogers, B.P.; Dempsey, R.J.; Meyerand, M.E. Diffusion Tensor MR Imaging in Diffuse Axonal Injury. American Journal of Neuroradiology 2002, 23, 794-802. Wang, J.; Hamm, R.J.; Povlishock, J.T. Traumatic Axonal Injury in the Optic Nerve: Evidence for Axonal Swelling, Disconnection, Dieback, and Reorganization. Journal of Neurotrauma 2011, 28, 1185-1198, doi:10.1089/neu.2011.1756. Cheng, C.L.Y.; Povlishock, J.T. The Effect of Traumatic Brain Injury on the Visual System: A Morphologic Characterization of Reactive Axonal Change. Journal of Neurotrauma 1988, 5, 47-60, doi:10.1089/neu.1988.5.47.

24. Turner, J.E.; Glaze, K.A. The early stages of Wallerian degeneration in the severed optic nerve of the newt (Triturus viridescens). Anat Rec 1977, 187, 291-310, doi:10.1002/ar.1091870303.

25. Erb, D.E.; Povlishock, J.T. Neuroplasticity following traumatic brain injury: a study of GABAergic terminal loss and recovery in the cat dorsal lateral vestibular nucleus. Exp Brain Res 1991, 83, 253-267, doi:10.1007/bf00231151.

26. Murray, B. Nerve Injury. In Encyclopedia of the Neurological Sciences (Second Edition), Aminoff, M.J., Daroff, R.B., Eds.; Academic Press: Oxford, 2014; pp. 333-335.

27. Chen, Y.-J.; Liang, C.-M.; Tai, M.-C.; Chang, Y.-H.; Lin, T.-Y.; Chung, C.-H.; Lin, F.-H.; Tsao, C.-H.; Chien, W.-C. Longitudinal relationship between traumatic brain injury and the risk of incident optic neuropathy: A 10-year follow-up nationally representative Taiwan survey. Oncotarget 2017, 8, 86924-86933, doi:10.18632/oncotarget.21008.

28. Yang, S.H.; Gustafson, J.; Gangidine, M.; Stepien, D.; Schuster, R.; Pritts, T.A.; Goodman, M.D.; Remick, D.G.; Lentsch, A.B. A murine model of mild traumatic brain injury exhibiting cognitive and motor deficits. J Surg Res 2013, 184, 981-988, doi:10.1016/j.jss.2013.03.075.

29. Schmued, L.C.; Hopkins, K.J. Fluoro-Jade B: a high affinity fluorescent marker for the localization of neuronal degeneration. Brain Res 2000, 874, 123-130.

30. Schneider, C.A.; Rasband, W.S.; Eliceiri, K.W. NIH Image to ImageJ: 25 years of image analysis. Nature methods 2012, 9, 671675.

31. Giolli, R.A.; Blanks, R.H.I.; Lui, F. The accessory optic system: basic organization with an update on connectivity, neurochemistry, and function. In Progress in Brain Research, Büttner-Ennever, J.A., Ed.; Elsevier: 2006; Volume 151, pp. 407440.

32. Horn, A.K.E.; Adamczyk, C. Chapter 9 - Reticular Formation: Eye Movements, Gaze and Blinks. In The Human Nervous System (Third Edition), Mai, J.K., Paxinos, G., Eds.; Academic Press: San Diego, 2012; pp. 328-366. 
33. Kozicz, T.; Bittencourt, J.C.; May, P.J.; Reiner, A.; Gamlin, P.D.R.; Palkovits, M.; Horn, A.K.E.; Toledo, C.A.B.; Ryabinin, A.E. The Edinger-Westphal nucleus: a historical, structural, and functional perspective on a dichotomous terminology. The Journal of comparative neurology 2011, 519, 1413-1434, doi:10.1002/cne.22580.

34. Morin, L.P.; Studholme, K.M. Retinofugal projections in the mouse. J Comp Neurol 2014, 522, 3733-3753, doi:10.1002/cne.23635.

35. RD, Z.; J., M.D. Neuroanatomy, Superior Colliculus. StatPearls [Internet] 2020.

36. Schröder, H.; Moser, N.; Huggenberger, S. The Mouse Caudate Putamen, Motor System, and Nucleus Accumbens. In Neuroanatomy of the Mouse: An Introduction; Springer International Publishing: Cham, 2020; pp. 305-318.

37. Seger, C. The visual corticostriatal loop through the tail of the caudate: circuitry and function. Frontiers in Systems Neuroscience 2013, 7, doi:10.3389/fnsys.2013.00104.

38. Yakushin, S.B.; Gizzi, M.; Reisine, H.; Raphan, T.; Büttner-Ennever, J.; Cohen, B. Functions of the nucleus of the optic tract (NOT). II. Control of ocular pursuit. Experimental brain research 2000, 131, 433-447, doi:10.1007/s002219900302.

39. Parhamifar, L.; Andersen, H.; Wu, L.; Hall, A.; Hudzech, D.; Moghimi, S.M. Chapter Twelve - Polycation-Mediated Integrated Cell Death Processes. In Advances in Genetics, Huang, L., Liu, D., Wagner, E., Eds.; Academic Press: 2014; Volume 88, pp. 353-398.

40. Kossmann, T.; Freedman, I.; Morganti-Kossmann, C. CHAPTER 104 - SPINAL TRAUMA. In Neurology and Clinical Neuroscience, Schapira, A.H.V., Byrne, E., DiMauro, S., Frackowiak, R.S.J., Johnson, R.T., Mizuno, Y., Samuels, M.A., Silberstein, S.D., Wszolek, Z.K., Eds.; Mosby: Philadelphia, 2007; pp. 1397-1408.

41. Maxwell, W.L. Damage to Myelin and Oligodendrocytes: A Role in Chronic Outcomes Following Traumatic Brain Injury? In Brain Sci; 2013; Volume 3, pp. 1374-1394.

42. Vaughn, J.E.; Pease, D.C. Electron microscopic studies of Wallerian degeneration in rat optic nerves. II. Astrocytes, oligodendrocytes and adventitial cells. Journal of Comparative Neurology 1970, 140, 207-225, doi:https://doi.org/10.1002/cne.901400205.

43. Lee, S.Y.; Chung, W.S. The roles of astrocytic phagocytosis in maintaining homeostasis of brains. J Pharmacol Sci 2021, 145, 223-227, doi:10.1016/j.jphs.2020.12.007.

44. Green, D.R.; Oguin, T.H.; Martinez, J. The clearance of dying cells: table for two. Cell Death \& Differentiation 2016, 23, 915926, doi:10.1038/cdd.2015.172.

45. Izzy, S.; Liu, Q.; Fang, Z.; Lule, S.; Wu, L.; Chung, J.Y.; Sarro-Schwartz, A.; Brown-Whalen, A.; Perner, C.; Hickman, S.E.; et al. Time-Dependent Changes in Microglia Transcriptional Networks Following Traumatic Brain Injury. Frontiers in cellular neuroscience 2019, 13, 307-307, doi:10.3389/fncel.2019.00307.

46. Yoo H, M.D. Neuroanatomy, Pupillary Light Reflexes and Pathway. Available online: https://www.ncbi.nlm.nih.gov/books/NBK553169/ (accessed on July 15, 2021).

47. Baver, S.B.; Pickard, G.E.; Sollars, P.J.; Pickard, G.E. Two types of melanopsin retinal ganglion cell differentially innervate the hypothalamic suprachiasmatic nucleus and the olivary pretectal nucleus. European Journal of Neuroscience 2008, 27, 17631770, doi:https://doi.org/10.1111/j.1460-9568.2008.06149.x.

48. Hill, C.S.; Coleman, M.P.; Menon, D.K. Traumatic Axonal Injury: Mechanisms and Translational Opportunities. Trends Neurosci 2016, 39, 311-324, doi:10.1016/j.tins.2016.03.002.

49. Wang, G.; Zhang, J.; Hu, X.; Zhang, L.; Mao, L.; Jiang, X.; Liou, A.K.; Leak, R.K.; Gao, Y.; Chen, J. Microglia/macrophage polarization dynamics in white matter after traumatic brain injury. J Cereb Blood Flow Metab 2013, 33, 1864-1874, doi:10.1038/jcbfm.2013.146.

50. Karve, I.P.; Taylor, J.M.; Crack, P.J. The contribution of astrocytes and microglia to traumatic brain injury. Br J Pharmacol 2016, 173, 692-702, doi:10.1111/bph.13125.

51. Cekanaviciute, E.; Fathali, N.; Doyle, K.P.; Williams, A.M.; Han, J.; Buckwalter, M.S. Astrocytic transforming growth factorbeta signaling reduces subacute neuroinflammation after stroke in mice. Glia 2014, 62, 1227-1240, doi:10.1002/glia.22675. 
52. Lööv, C.; Hillered, L.; Ebendal, T.; Erlandsson, A. Engulfing astrocytes protect neurons from contact-induced apoptosis following injury. PLoS One 2012, 7, e33090, doi:10.1371/journal.pone.0033090.

53. Cekanaviciute, E.; Buckwalter, M.S. Astrocytes: Integrative Regulators of Neuroinflammation in Stroke and Other Neurological Diseases. Neurotherapeutics 2016, 13, 685-701, doi:10.1007/s13311-016-0477-8.

54. Wang, G.; Zhang, J.; Hu, X.; Zhang, L.; Mao, L.; Jiang, X.; Liou, A.K.-F.; Leak, R.K.; Gao, Y.; Chen, J. Microglia/Macrophage Polarization Dynamics in White Matter after Traumatic Brain Injury. Journal of Cerebral Blood Flow E Metabolism 2013, 33, 1864-1874, doi:10.1038/jcbfm.2013.146.

55. Huang, X.; You, W.; Zhu, Y.; Xu, K.; Yang, X.; Wen, L. Microglia: A Potential Drug Target for Traumatic Axonal Injury. Neural Plasticity 2021, 2021, 5554824, doi:10.1155/2021/5554824.

56. Wylot, B.; Mieczkowski, J.; Niedziolka, S.; Kaminska, B.; Zawadzka, M. Csf1 Deficiency Dysregulates Glial Responses to Demyelination and Disturbs CNS White Matter Remyelination. Cells 2019, 9, doi:10.3390/cells9010099.

57. Patel, V.C.; Jurgens, C.W.D.; Krahe, T.E.; Povlishock, J.T. Adaptive reorganization of retinogeniculate axon terminals in dorsal lateral geniculate nucleus following experimental mild traumatic brain injury. Experimental Neurology 2017, 289, 8595, doi:https://doi.org/10.1016/j.expneurol.2016.12.012.

58. Kimbler, D.E.; Shields, J.; Yanasak, N.; Vender, J.R.; Dhandapani, K.M. Activation of P2X7 Promotes Cerebral Edema and Neurological Injury after Traumatic Brain Injury in Mice. PLoS One 2012, 7, e41229, doi:10.1371/journal.pone.0041229.

59. Wang, Y.C.; Cui, Y.; Cui, J.Z.; Sun, L.Q.; Cui, C.M.; Zhang, H.A.; Zhu, H.X.; Li, R.; Tian, Y.X.; Gao, J.L. Neuroprotective effects of brilliant blue $G$ on the brain following traumatic brain injury in rats. Mol Med Rep 2015, 12, 2149-2154, doi:10.3892/mmr.2015.3607. 